\title{
Infografia na web para a inclusão digital de alunos surdos
}

\author{
Mariana Lapolli, Universidade Federal de Santa Catarina - UFSC, \\ marilapolli@gmail.com \\ Tarcísio Vanzin, UFSC, tvanzin@gmail.com \\ Vania Ribas Ulbricht, UFSC, vrulbricht@gmail.com
}

\section{Resumo}

Os conteúdos na web, geralmente, não são elaborados para pessoas que possuem algum tipo de necessidade especial. No caso dos surdos, uma das dificuldades a serem enfrentadas é a diversidade de opções linguísticas que essas pessoas utilizam. Como esse grupo se orienta a partir da visão, parte-se do principio que materiais com forte apelo visual possa ser interessante para eles. Diante desse pressuposto, infografias na web voltadas para surdos foram desenvolvidas para o projeto "Educação Inclusiva: Ambiente Web acessível com Objetos de Aprendizagem para Representação Gráfica", com apoio da CAPES e CNPq, cujo objetivo é desenvolver um ambiente web adaptável e acessível para representação gráfica na ótica da educação inclusiva. Neste artigo é apresentada uma dessas infografias, sendo que o objetivo é verificar a infografia desenvolvida para a inclusão digital de alunos surdos. Esta verificação foi realizada com um grupo de pessoas surdas que acessaram a infografia e as atividades relativas ao seu conteúdo e, posteriormente, participaram de entrevistas semiestruturadas, manifestando suas necessidades e preferências em relação à forma de apresentação dos conteúdos. Como resultado, esta pesquisa contribui para a inclusão digital de alunos surdos, mostrando-se relevante pelo aspecto social que envolve.

Palavras-chaves: infografia na web, inclusão digital, surdos.

\section{Infographic on the web for digital inclusion of deaf students}

\begin{abstract}
The webs contents are generally not designed for people who have some kind of special need. In the case of deaf individuals, one of the difficulties to be faced is the diversity of language options that these people use. As this grup is visually oriented, it is assumed that materials with atrong visual appeal would be of interest to them. Given this assumption, infographics on the web facing deaf were developed for the Project "Inclusive Education: Affordable Web Environment with Learning Objects for Graphic Representation", with support from CAPES and CNPq, whose goal is to develop na adaptable and accessible environment for web representation graphics from the perspective of inclusive education. This article presentes one of these infographics and the goal is to verify infographics designed for the digital inclusion of deaf students. This verification was conducted with a group of deaf people who accessed the infographic and activities concerning their content, and later participated in semi-structured interview, expressing their needs ans preferences regarding the presentation of contente. As a result, this research contributes to the digital inclusion of deaf studentes, and is therefore relevant for the social aspect that involves.
\end{abstract}

Keywords: infographic on the web, digital inclusion, deaf. 


\section{Introdução}

A inclusão diz respeito à acessibilidade, fundamental para a concessão de um acesso às informações de forma autônoma para todas as pessoas, independente de suas características. "A não-observância da acessibilidade no espaço digital pelos autores dos materiais disponibilizados nessa forma pode ser considerada como uma discriminação feita a milhares de usuários" (Torres et al., 2002, p.85).

No Brasil existem 9.722.163 de pessoas com deficiência auditiva, sendo que 347.481 são surdos (IBGE, 2010). Para que estas pessoas tenham possibilidades de acesso aos conteúdos educativos na web, algumas medidas são necessárias, conforme já apontado por diversos autores que estudam esta temática (Martins, 2005; Corradi, Vidotti, 2007; Farjardo et al., 2008; Al-Bayati, Hussein, 2010; Debevc et al. 2010; Bottoni et al., 2013; Nordin et al., 2013; Saito et al., 2013). Neste sentido, o World Wide Web Consortium - W3C, um consórcio internacional que desenvolve padrões e diretrizes para a web, tem o seu foco na redução de barreiras existentes na web, no entanto "as diretrizes existentes no W3C são de cunho geral, focadas na web, e embora muito úteis não são específicas para usuários surdos" (Abreu et al., 2010, p. 490).

$\mathrm{Na}$ elaboração de conteúdos web para surdos é preciso levar em consideração a heterogeneidade deste grupo, sobretudo em relação à sua opção linguística e à competência nessas línguas. Sobre esse assunto, Souza (2000) revela que os surdos possuem dificuldade em ler textos normais. A causa disto é explicada pela limitação nas trocas de expressões com os ouvintes, o que reduz o vocabulário, assim como pelo fato da língua de sinais possuir uma sintaxe diferenciada da língua escrita. Isto pode ser considerado um problema, uma vez que a língua falada e escrita ocupa um lugar de relevância na produção do conhecimento (Campello, 2007).

Guimarães (2009) e Rosa e Luchi (2010) defendem o uso de imagens para transmitir conhecimentos para os surdos. Neste sentido, em sua tese, Lapolli (2014) propôs a utilização de infografia na web, caracterizada pela associação de imagens com textos curtos e objetivos, como ferramenta didática para os alunos surdos. Durante essa pesquisa, que é pertinente ao Projeto CAPES-AUX-PROESP 1026/2009, foi desenvolvida uma infografia na web que apresenta conceitos relativos aos fundamentos da Geometria Descritiva - GD, bem como conceitos relacionados à projeção cilíndrica ortogonal.

Diante do exposto, o intuito deste artigo é verificar a infografia desenvolvida para a inclusão digital de alunos surdos. Para isto, um grupo de pessoas surdas acessaram a infografia e, posteriormente, participaram de entrevistas semiestruturadas, manifestando suas necessidades e preferências em relação a forma de apresentação dos conteúdos. Como resultado, esta pesquisa abre oportunidades para o desenvolvimento pessoal do surdo, contribuindo para a inclusão digital deste público. Mostra-se, assim, relevante pelo aspecto social que envolve.

\section{Inclusão digital de alunos surdos}

A inclusão está categoricamente ligada à acessibilidade (Monteiro, 2009). No espaço digital, acessibilidade "consiste em tornar disponível ao usuário, de forma autônoma, toda a informação que lhe for franqueável [...], independentemente de suas características corporais [...]" (Torres et al., 2002, p.85). No contexto da educação, a acessibilidade na web implica no desenvolvimento de ambientes virtuais que, por meio de processos educacionais e tecnologias facilitadoras, atendam as demandas de seus usuários.

Para o usuário surdo, de acordo com Martins (2005), é necessário criar páginas que privilegiem a língua de sinais e a imagem. Deste modo, os textos devem ser curtos e 
concisos, a navegação deve ser clara e os vídeos com o intérprete da língua de sinais devem aparecer no lado esquerdo da tela (Debevc et al., 2010). Corradi e Vidotti (2007) propõem, além do uso de vídeos com conteúdos informacionais traduzidos para a língua de sinais, o uso de legendas para vídeos com áudio escritas em português.

Para Farjardo et al. (2008), a interface gráfica (hipertexto com links gráficos) pode facilitar a performance dos surdos comparada a interface verbal (hipertexto com links verbais), reduzindo a desvantagem de acesso ao conhecimento em relação aos ouvintes. Isto é evidenciado na pesquisa de Saito et al. (2013) que aponta que os surdos, mesmo com um alto nível de educação, possuem dificuldade de ler textos longos e de navegar nos menus baseados em textos. Esses autores enfatizam a necessidade de recursos como a Língua de Sinais e o SignWriting - SW, que é uma das formas de escrita de sinais mais bem aceita pelos surdos.

Bottoni et al. (2013) sugerem o desenvolvimento de um ambiente virtual voltado para adultos surdos, cujos princípios sejam de evitar ao máximo, os textos e optar por uma apresentação visual da informação. Neste sentido, Nordin et al. (2013) afirmam que com a presença de mais gráficos visuais nos materiais de aprendizagem, os estudantes surdos podem aprender e ter uma melhor performance, em comparação a textos longos.

No ambiente digital, diversas formas de apresentação dos conteúdos podem ser exploradas. Sobre este tema, Al-Bayati e Hussein (2010) dizem que os ambientes hipermídias suportam essas diversas maneiras de se comunicar, sendo adequados para a aprendizagem do surdo.

\section{Infografia na web desenvolvida}

Tendo em vista a competência visual dos surdos e a preferência por textos curtos, Lapolli (2014) propôs a utilização de infografia na web como ferramenta didática para este público. A infografia na web aproveita as possibilidades de integração de recursos que incluem textos, ícones (próprios da simbologia e do grafismo), elementos visuais (na fotografia, presentes na televisão e no cinema) e sons (silencio e ruídos), bem como ferramentas informáticas e programas apropriados (Marín Ochoa, 2009). Rodrigues (2009, p.35) complementa essa ideia ao dizer que "indo além da infografia impressa, a infografia interativa no ciberespaço utiliza um conjunto de elementos multimídia (áudio, vídeo, fotos e imagens em movimento) que forma uma linguagem caracterizada pela multilinearidade".

A capacidade das infografias de transmitir informações e gerar conhecimentos por meio de representações verbais e visuais faz com que este recurso tenha potencialidade no âmbito educacional. Nesta direção, Valero Sancho (2012) afirma que a infografia é uma das melhores formas de ensinar devido às múltiplas ferramentas que podem ser utilizadas a seu serviço, conduzindo os estudantes pelos caminhos da aprendizagem.

Segundo Bottentuit Junior et al. (2011) a utilização de infografias promove uma aprendizagem onde os alunos podem se deparar com uma realidade mais consistente. Andrade (2011, p.66) declara que

"[...] a infografia busca uma aproximação do estudante ao objeto de estudo, provocando uma experiência interativa mais profunda e significativa, trazendo uma informação muitas vezes distante da realidade de todos, de uma forma clara e compreensível".

Buscando aproximar o conteúdo, neste caso sobre os fundamentos da GD e sobre projeção cilíndrica ortogonal, à realidade dos alunos foi desenvolvida uma infografia na web utilizando a metáfora dos objetos presentes num escritório de arquitetura. A tela inicial (Figura 1) desta infografia contém objetos que são links para 


CINTED-UFRGS Novas Tecnologias na Educação

os conteúdos que podem ser acessados de maneira não linear pelos usuários. Esses objetos possuem um efeito de brilho para que os alunos saibam onde é possível clicar.

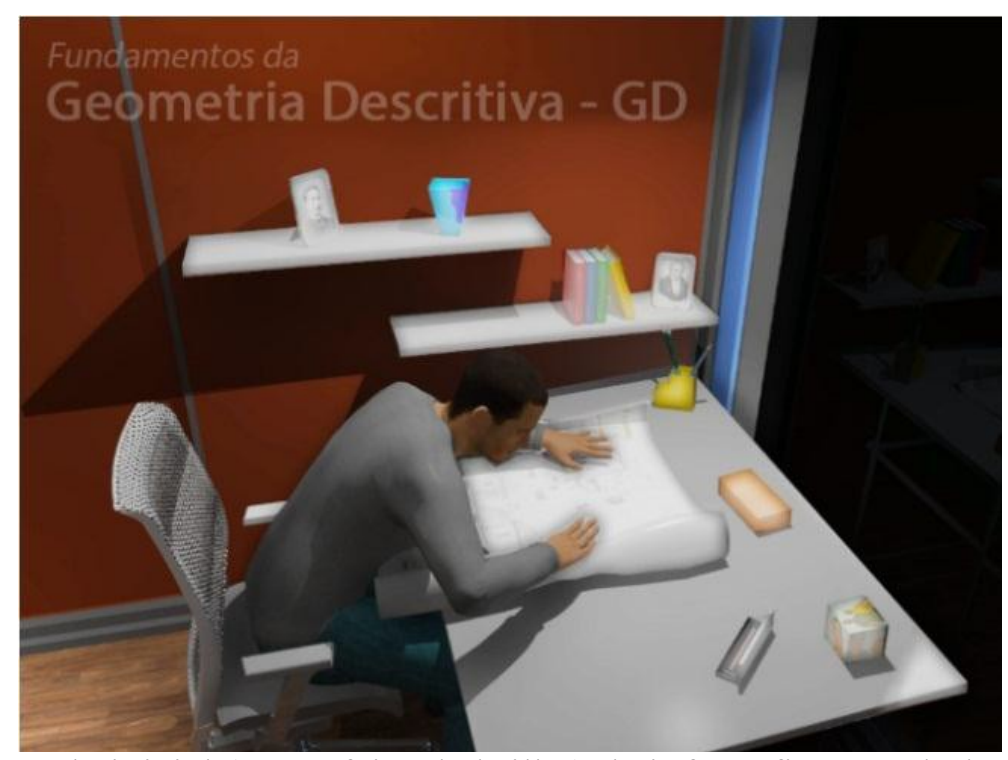

Figura 1 - Tela inicial (com efeito de brilho) da infografia na web desenvolvida

Cada vez que o aluno clica num objeto surge, sobre a tela principal, uma tela com leve transparência onde os conteúdos são apresentados (Figura 2). Sempre os conteúdos escritos e/ou as imagens estão posicionados à direita da tela, enquanto que a tradução em língua de sinais ou em SW permanecem à esquerda. $\mathrm{O}$ aluno pode acessar uma ou outra tradução clicando nos ícones que aparecem destacados no círculo vermelho nas figuras 3 e 4 .

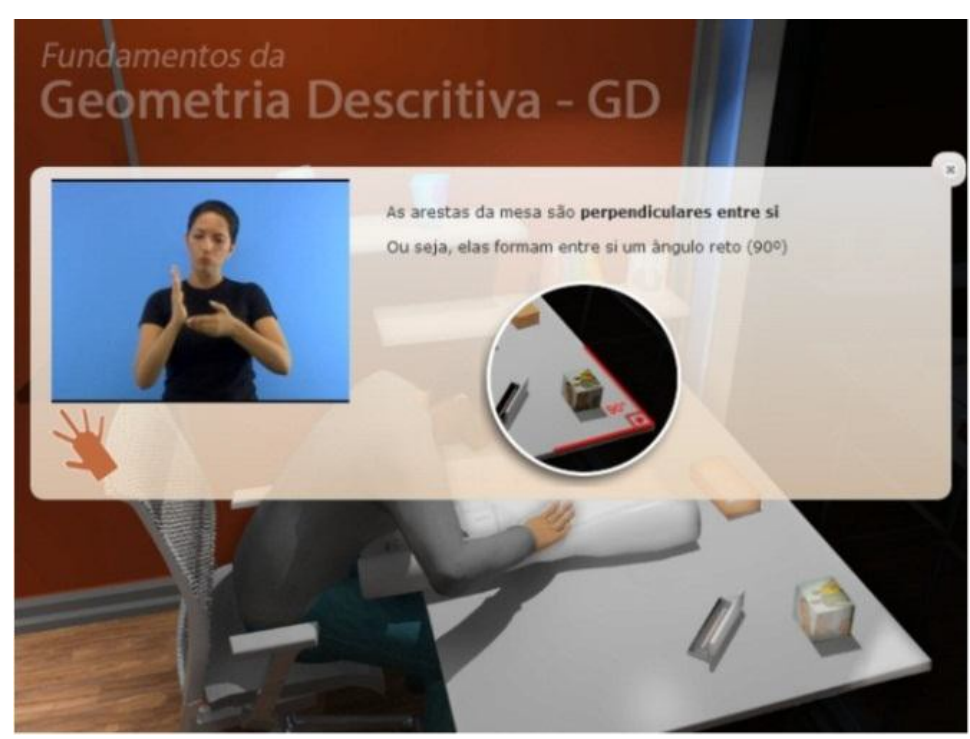

Figura 2 - Exemplo de tela de conteúdo

$\mathrm{Na}$ prateleira da tela inicial existem quatro livros. Quando um deles é clicado, abre uma tela (Figura 3) com a capa e o título à direita. À esquerda, tem uma explicação sucinta do conteúdo tratado no livro, assim como a tradução em LIBRAS e SW. Ao abrir o livro (Figura 4), os conteúdos, em textos, imagens e animações sobre GD também aparecem dispostos sempre à direita e as traduções à esquerda. Esse padrão de posicionamento dos elementos foi adotado em toda a infografia com o intuito de que os 
alunos pudessem facilmente identificar a localização dos conteúdos e suas traduções cada vez que mudassem de tela.

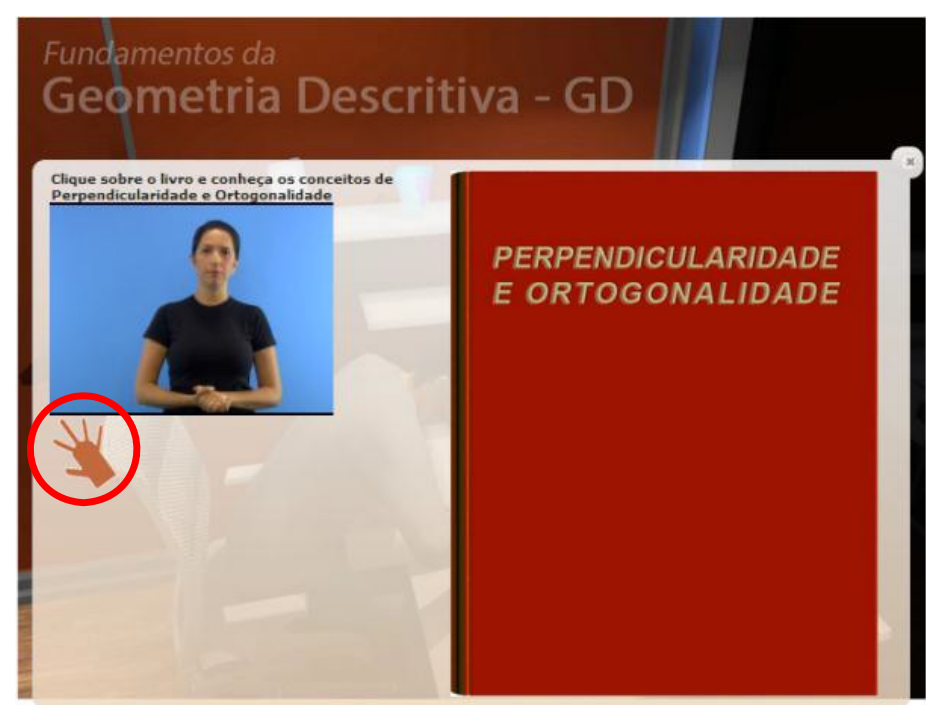

Figura 3 - Exemplo de Tela com a capa do livro

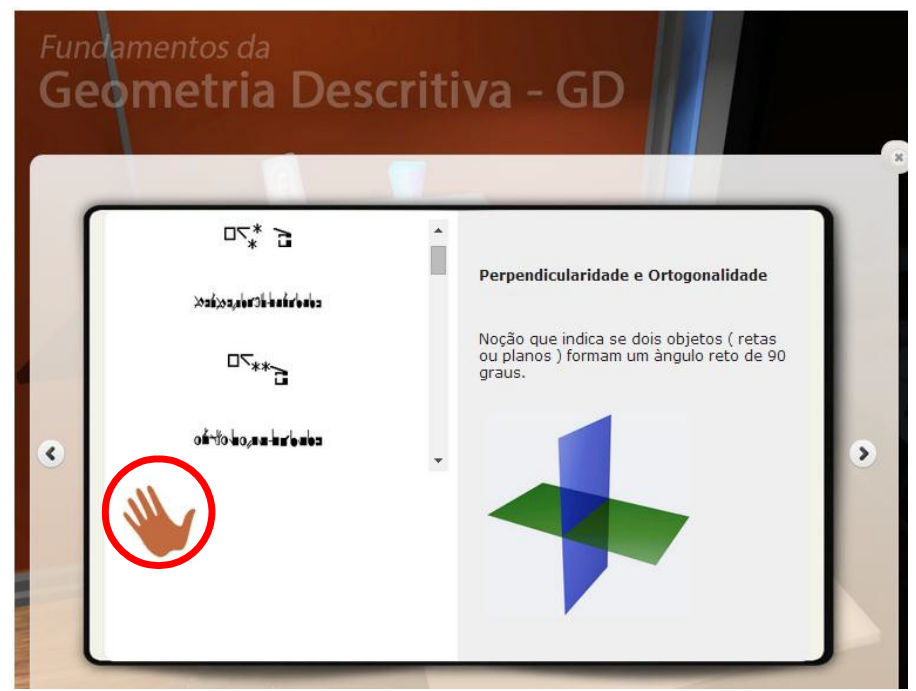

Figura 4 - Exemplo de Tela com o livro aberto

Para verificar os conhecimentos adquiridos pelos alunos, atividades de múltipla escolha relativas ao conteúdo apresentado foram elaboradas. Estes exercícios apresentam um enunciado traduzido em língua de sinais e, como opções de respostas, imagens a serem escolhidas pelo estudante. Na Figura 5 é possível verificar um exemplo de questão. 


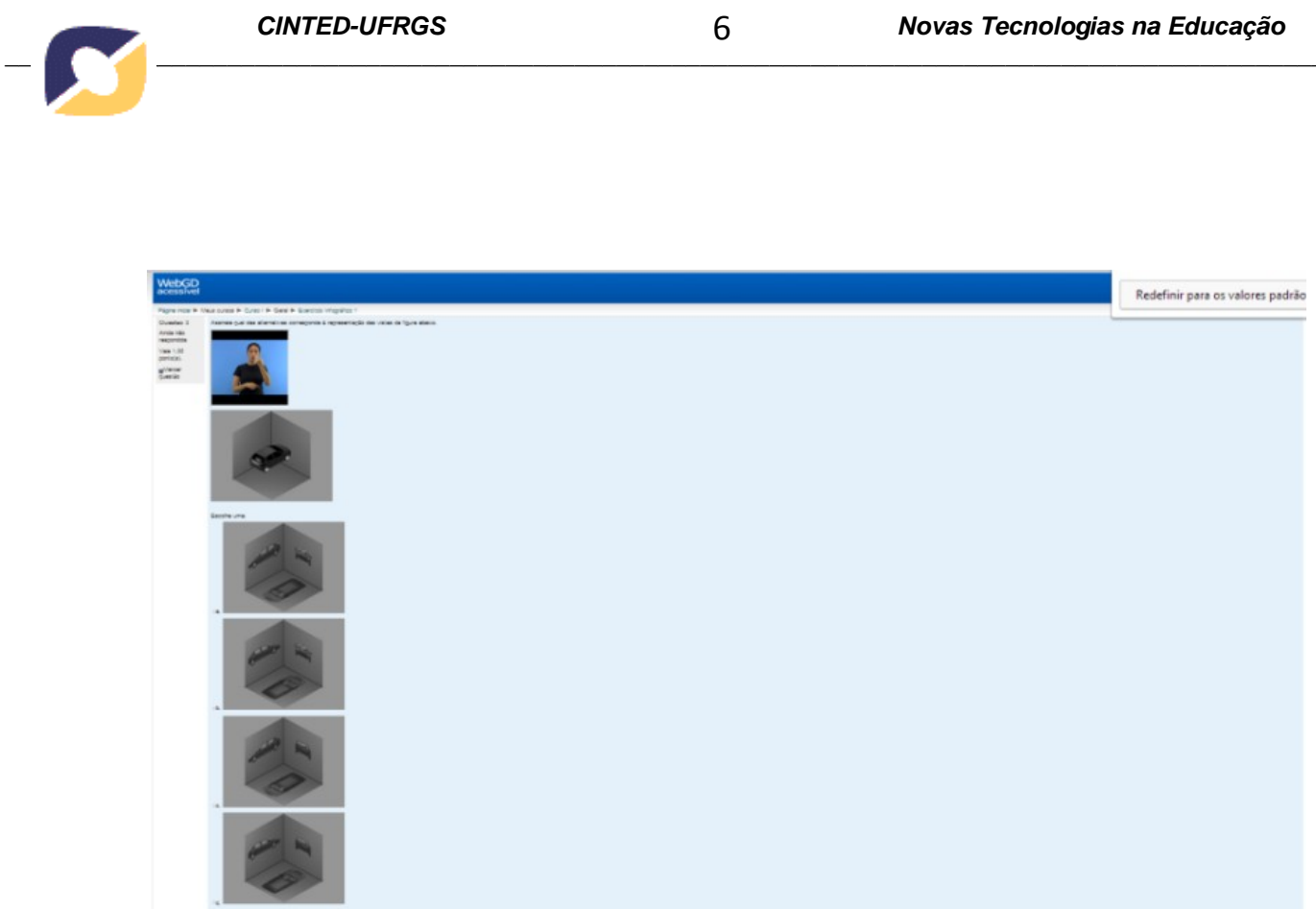

Figura 5 - Exemplo de exercício com opções de respostas

\section{Verificação da infografia desenvolvida para a inclusão digital de alunos surdos}

A verificação da infografia desenvolvida foi realizada com 12 pessoas surdas que estão acostumados a acessarem ambientes virtuais. Da totalidade de participantes, que expuseram suas necessidades e preferências em relação a forma de apresentação dos conteúdos, 11 são graduandos e um é pós-graduando, o que demonstra que essas pessoas possuem um elevado nível educacional.

Para extrair a opinião dos participantes da pesquisa utilizou-se o método de entrevistas semiestruturada, que se trata de uma conversa a dois, realizada por iniciativa do entrevistador, com o desígnio de fornecer informações pertinentes a um objeto de pesquisa (Minayo, 1993). Assim, um roteiro de entrevista foi estrategicamente formulado de maneira que as respostas instituíssem o melhor acesso possível às representações sociais.

Por meio de perguntas fechadas e abertas, os entrevistados discorreram sobre o tema proposto, logo após acessarem a infografia desenvolvida e realizarem as atividades propostas. Cada entrevista foi gravada em vídeo e posteriormente, transcrita. Como os participantes da pesquisa são pessoas surdas, foi necessária a presença de uma intérprete de LIBRAS durante a entrevista. Ela, também, verificou a transcrição, comparando-as com os vídeos gravados para corrigir possíveis falhas de tradução.

A partir das entrevistas é possível perceber que, para os participantes da pesquisa, a infografia na web desenvolvida é criativa, organizada, muito visual, chama a atenção, desperta a curiosidade e representa uma forma inovadora para a aprendizagem. Os surdos destacaram sua preferência pelas imagens, seja qual for o tipo (fotos, ilustrações 2D, ilustrações 3D etc.), sendo que o importante é haver um equilíbrio no uso dessas imagens, não poluindo o ambiente. Corrobora, neste sentido, com Costa, Tarouco e Biazus (2011) que dizem que, ao escolher e criar imagens para conteúdos educacionais é preciso ter cuidado para não sobrecarregar a carga cognitiva do estudante com imagens meramente decorativas que não contribuem para a compreensão do conteúdo.

Em relação à navegação, os participantes foram, aos poucos, se adaptando ao ambiente. "No começo eu fiquei um pouco confuso, mas eu vi que brilhava e já entendi. Se não tivesse nada, eu não saberia onde clicar", afirmou um deles. Assim, eles consideraram atraente ver os objetos piscando na tela inicial, ficando claro onde eles deveriam clicar. “[...] os objetos estavam brilhando [...] e eu pensei: „deixa eu ver o que

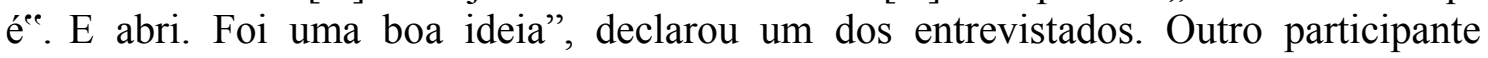


comentou: "tem várias coisas para serem acessadas e tinha a ver com o desenho e o contexto".

A não linearidade foi considerada uma característica interessante por possibilitar escolhas, porém fez com que algumas pessoas se sentissem perdidas. Esses indivíduos revelaram que gostariam de um direcionamento, apontando em qual sequência os conteúdos deveriam ser acessados (do básico ao mais complexo).

Os surdos afirmaram ainda que a imagem facilita o aprendizado e combina perfeitamente com seu sistema visual. Além disso, destacaram a necessidade de utilização de textos curtos, uma vez que a maioria dos surdos tem dificuldades de entender o português. A dificuldade que os surdos possuem em relação a produção e compreensão da língua escrita foi mencionada por Bottoni et al. (2013) Nordin et al. (2013) na revisão bibliográfica desta pesquisa. Deste modo, de acordo com as opiniões coletadas, a imagem associada a textos curtos possibilita o acesso a uma informação completa, sendo que o ideal é diminuir o máximo possível a quantidade de textos em português.

Sobre o uso de vídeos com a tradução do texto para a língua de sinais, uma das pessoas que acessaram a infografia desenvolvida afirmou: "se eu não entendia a palavra em português, eu olhava o vídeo de novo para associar [...] É como se fosse um suporte". O tamanho da tela de vídeo e a cor da legenda foram aprovados pelos participantes. A legenda contribuiu para a compreensão do significado de diversas palavras, contudo os surdos sugeriram que ela fosse utilizada junto a um sinal que represente determinada palavra e não com sua soletração em língua de sinais, como foi realizado na infografia acessada por eles. Além disso, os entrevistados alertaram para o fato de que algumas palavras longas foram soletradas diversas vezes, dizendo que somente na primeira vez seria o suficiente e nas demais o ideal seria encontrar um sinal que representasse essas palavras. Tendo em vista que, devido a não linearidade do ambiente, não seria possível saber qual vídeo o usuário veria pela primeira vez, a solução proposta foi a criação de um glossário. Desta maneira, os usuários poderiam recorrer à explicação de uma determinada palavra ou termo em língua de sinais.

O SW não foi muito utilizado, pois a maioria dos participantes não sabia ler a escrita de sinais: "Escrita de sinais é um sistema novo ainda [...] Poucas pessoas sabem ler", declarou um dos estudantes. Contudo, na opinião dos surdos, é importante difundir essa forma de escrita.

Quanto às atividades, os participantes alegaram que seria melhor se elas estivessem embutidas na própria infografia para que os conceitos não fossem esquecidos no momento de responder as questões. Para eles, foi bom receber o feedback das suas respostas (que eram assinaladas em verde ou vermelho quando eles acertavam ou erravam). Faltaram apenas explicações para seus erros e acertos: "a gente fica querendo saber por que está errado, por que está certo".

De acordo com os entrevistados, por meio da infografia na web os conteúdos são apresentados de uma forma bem didática e divertida, fazendo com que a disciplina estudada pareça mais simples. Como a matéria é passada por etapas e todos os textos estão traduzidos (em língua de sinais e SW), fica fácil de entender e fixar o conteúdo. "Eu achei fantástico. Eu realmente fiquei admirada como eu consegui aprender algo tão difícil”, revelou uma entrevistada.

\section{Conclusões}

As referências bibliográficas sobre a inclusão digital de alunos surdos e sobre infografia auxiliaram no desenvolvimento de um objeto que utiliza como metáfora um escritório de arquitetura. Nesta infografia, buscou-se a acessibilidade dos surdos por meio de uma 
apresentação bastante visual e com poucos textos. Além disso, todos os textos foram traduzidos para a língua de sinais, uma vez que os artigos citados nesta pesquisa apontavam a necessidade dessa tradução para os surdos, bem como para o SW que, conforme enfatizado, se trata de uma das formas de escrita de sinais mais bem aceita por esse público.

Para garantir a acessibilidade, além das traduções, é necessário que a equipe de desenvolvimento esteja atenta a aspectos relativos à escolha e organização das imagens utilizadas, à navegação, à forma como será realizado o vídeo com a tradução para a língua de sinais (legendas, sinalização, soletração, etc.), à forma como serão apresentadas as atividades e seus resultados, entre outros. Cada detalhe foi discutido pelos participantes dessa pesquisa, que são pessoas surdas habituadas a acessarem ambientes virtuais e que possuem um elevado nível educacional.

Para os entrevistados, as infografias são uma forma interessante de apresentação de conteúdos para as pessoas surdas devido ao forte apelo visual, que combina com o sistema visual do surdo, e a utilização de textos curtos. Esta ferramenta pode ser utilizada para o ensino e aprendizagem na web, sendo bastante criativo e atrativo para os alunos.

As opiniões dos usuários surdos, extraídas pelas entrevistas, visaram verificar a infografia desenvolvida para o projeto CAPES-AUX-PROESP 1026/2009, no entanto, as apreciações coletadas podem auxiliar no desenvolvimento de futuras infografias na web para surdos com conteúdos relativos a qualquer área do conhecimento. Desta maneira, milhares de pessoas podem ser beneficiadas por uma experiência educativa que contribui para a inclusão digital dos surdos.

\section{Agradecimentos}

À Coordenação de Aperfeiçoamento de Pessoal de Nível Superior - CAPES pelo apoio no subsídio desta pesquisa. Ao grupo de pessoas surdas que participaram desta pesquisa.

\section{Referências bibliográficas}

ABREU, P. M. ; PRATES, R. O. ; BERNARDINO, E. L. A. Recomendações de acessibilidade para projetos de TICs para alfabetização de crianças surdas. In: Seminário Integrado de Software e Hardware, 37., 2010, Porto Alegre. Anais. Porto Alegre: SBC, 2010, p. 1-15.

AL-BAYATI, M. A.; HUSSEIN, K. Q. Evaluating the efficiency of the instructional websites "which are oriented to the deaf students" according to the technical criteria. Journal of Convergence Information Technology, Korea, v.5, n.2, abr. 2010.

ANDRADE, R. de O. Infografia educacional: uma análise sobre seu potencial pedagógico e aplicação no ensino. Paraná: Programa de Pós-Graduação em Design Informacional/PUCPR, 2011. 93p. Monografia.

BOTTENTUIT JUNIOR, J. B.; LISBOA, E. S.; COUTINHO, C. P. O infográfico e as suas potencialidades educacionais. In: Encontro Nacional de Hipertexto e Tecnologias Educacionais, 4., 2011, Sorocaba. Anais. Sorocaba: set. 2011.

BOTTONI, P.; BORGIA, F. BUCCARELLA, D.; CAPUANO, D.; MARSICO, M. de; LABELLA, A. Stories and signs in an e-learning environment for deaf people. International Journal of Universal Access in the Information Society. V.12, n.4, p 369-386, 2013. 
CAMPELLO, A.R. e S. Pedagogia Visual / Sinal na educação dos surdos. In: QUADROS, R.M. de; PERLIN, G. (org.) Estudos surdos II. Petrópolis: Arara Azul, 2007. p100-131.

CORRADI, J.A. M.; VIDOTTI, S. A. B. G. Arquitetura da informação para ambientes informacionais digitais inclusivos: acessibilidade para minorias lingüísticas surdas. In: Encontro Nacional de Ensino e Pesquisa da Informação, 7., 2007, Salvador. Anais. Salvador: 04-06 jun 2007. Disponível em: $<$ http://dici.ibict.br/archive/00001106/01/arquiteturadainforma\%C3\%A7\%C3\%A3opara ambientes.pdf $>$. Acesso em: 10 out. 2011.

COSTA, V. M. da; TAROUCO, L. M. R.; BIAZUS, M. C. V. Criação de Objetos de Aprendizagem baseados em infográficos. 2011. Disponível em: $<$ http://laclo2011.seciu.edu.uy/publicacion/laclo/laclo2011_submission_68.pdf $>$. Acesso em: 10 fev. 2013.

DEBEVC, M.; KOSEC, P.; HOLZINGER, A. E-learning accessibility for the deaf and hard of hearing - Practical examples and experiences. LNCS 6389. p 203-213, 2010.

FARJARDO, I.; ARFÉ, B.; ALTOÉ, G.; BENEDETTI, P. Hyperlink Format, Categorization Abilities and Memory Span as Contributors to Deaf Users Hypertext Access. Journal of Deaf Studies and Deaf Education, Reino Unido. v. 13, n.1, p 87102, dez. 2008.

GUIMARÃES, A.D.S. Leitores surdos e acessibilidade virtual mediada por tecnologias de informação e comunicação. Cuiabá: IFMS, 2009. 71p. Trabalho de Conclusão Curso.

IBGE. Instituto Brasileiro de Geografia e Estatística. 2010. Disponível em: $<$ http://www.censo2010.ibge.gov.br/>. Acesso em: 15 jan. 2012.

LAPOLLI, M. Visualização do conhecimento por meio de narrativas infográficas na web voltadas para surdos em comunidades de prática. Florianópolis: UFSC, 2014. 276p. Tese de Doutorado.

MARÍN OCHOA, B.E. La infografía digital, una nueva forma de comunicación. Barcelona: Faculdad de Ciencias de la Comunicación/UAB, 2009. 506p. Tese de Doutorado.

MARTINS, E. Cultura surda, educação e novas tecnologias em Santa Catarina. Florianópolis: UFSC, 2005. Dissertação de Mestrado.

MINAYO, M. C.de S. O desafio do conhecimento. São Paulo: Hucitec,1993.

MONTEIRO, R. Acessibilidade e Inclusão Digital: práticas de e-learning nas universidades públicas portuguesas. 2009. Disponível em: $<$ www.aminharadio.com/gtaedes/sites/default/files/apresent_rmonteiro.pdf $>$. Acesso em: 20 jul. 2011. 
NORDIN, N. M.; ZAHARUDIN, R.; YASIN, M. H. M.; DIN, R.; EMBI, M. A; LUBIS, M. A. ICT in education for deaf learners: Teachers' perspective. Research Journal of Applied Sciences. v. 8, p103-111, 2013.

RODRIGUES, A. V. Infografia Interativa em base de dados no jornalismo digital. Salvador: Pós-Graduação em Comunicação e Cultura Contemporâneas/UFBA, 2009. 130p. Dissertação de Mestrado.

ROSA, E. F.; LUCHI, M. Semiótica imagética: a importância da imagem na aprendizagem. In: ENCONTRO DO CELSUL, 9., 2010, Palhoça. Anais. Palhoça: out. 2010 .

SAITO, D. S.; PIVETTA, E. M.; ULBRICHT, V. R.; MACEDO, C. M. S. Evaluation of accessibility with the deaf user. Communications in Computer and Information Science. v. 373, p. 276-280, 2013.

SOUZA, G. C. de. Modelo de Aprendizado Cooperativo para Surdos Baseado em Ambiente Computacional (MACS). Florianópolis: PPGEP/UFSC, 2000. 109p. Dissertação de Mestrado.

TORRES, E. F.; MAZZONI, A. A.; ALVES, J. B. da M. A acessibilidade à informação no espaço digital. Ci. Inf. Brasília, v. 31, n. 3, p. 83-91, set./dez. 2002. Disponível em: <www.scielo.br/pdf/ci/v31n3/a09v31n3.pdf>. Acesso em: 01 out. 2011.

VALERO SANCHO, J. L. Infografía Digital: la visualización sintética. Barcelona: Boch, 2012. 238 p. 